\title{
Reconstruction of the spontaneously broken gauge theory in non-commutative geometry
}

\author{
Yoshitaka OKUMURA円, \\ Department of Natural Science, Chubu University, Kasugai 487, Japan \\ and \\ Katsusada MorITA \\ Department of Physics, Nagoya University, Nagoya 464-01, Japan
}

\begin{abstract}
The scheme previously proposed by the present authors is modified to incorporate the strong interaction by affording the direct product internal symmetry. We do not need to prepare the extra discrete space for the color gauge group responsible for the strong interaction to reconstruct the standard model and the left-right symmetric gauge model(LRSM ). The approach based on non-commutative geometry leads us to presents many attractive points such as the unified picture of the gauge and Higgs field as the generalized connection on the discrete space $M_{4} \times Z_{N}$. This approach leads us to unified picture of gauge and Higgs fields as the generalized connection. The standard model needs $N=2$ discrete space for reconstruction in this formalism. LRSM is still alive as a model with the intermediate symmetry of the spontaneously broken $\mathrm{SO}(10)$ grand unified theory(GUT). $N=3$ discrete space is needed for the reconstruction of LRSM to include two Higgs bosons $\phi$ and $\xi$ which are as usual transformed as $\left(2,2^{*}, 0\right)$ and $(1,3,-2)$ under $\mathrm{SU}(2)_{L} \times \mathrm{SU}(2)_{R} \times \mathrm{U}(1)_{Y}$, respectively. $\xi$ is responsible to make $\nu_{R}$ Majorana fermion and so well explains the seesaw mechanism. Up and down quarks have the different masses through the vacuum expectation value of $\phi$.
\end{abstract}

\footnotetext{
${ }^{1}$ e-mail address: okum@isc.chubu.ac.jp

${ }^{2}$ e-mail address: h44753a@nucc.cc.nagoya-u.ac.jp
} 


\section{Introduction}

Since Connes [1] has proposed the idea to understand the Higgs mechanism based on noncommutative geometry (NCG) in the discrete space $M_{4} \times Z_{2}$, several different versions have appeared to make NCG approach more applicable and understandable, and many works have been done so far $[2] \sim 9$.

One of versions has been initiated by Sitarz [3] in a way more familiar with the ordinary differential geometry. The present authors [7] have developed the formalism to be able to apply to the gauge theory with complex symmetry breaking pattern by introducing the more fundamental fields $a_{i}(x, n)$ and the matrix function $M_{n m}$, where $x$ is the variable in ordinary Minkowski space $M_{4}, i$ is a variable in the extra internal space and $n, m$ are the arguments in the discrete space $Z_{N}$. Gauge fields are composed of $a_{i}(x, n)$, which is similar to the effective gauge field in Berry phase [10]. This may provide a key to explore the extra internal space with the variable $i$. The matrix $M_{n m}$ determines the scale and pattern of the spontaneous breakdown of gauge symmetry and composes the Higgs field along with $a_{i}(x, n)$. Thus, in our formalism Higgs fields are created at the same time as the spontaneous breakdown of symmetry takes place, which leads to the quite different viewpoint of Higgs mechanism from the ordinary one. Then, the unified picture of the gauge fields and Higgs fields as the generalized connection in NCG on the discrete space $M_{4} \times Z_{N}$ is realized.

According to our formalism [7] we could reconstruct the standard model [6], [7], $\mathrm{SU}(5)$ grand unified theory (GUT) [7] and SO(10) GUT [11]. The color SU(3) gauge group responsible for the strong interaction is well incorporated in the reconstructions of SU(5) and $\mathrm{SO}(10)$ GUTs from the outset. However, in the case of the standard model [6], [7] the extra sheet of the discrete space is needed to incorporate the color SU(3) gauge group, which makes the reconstruction of the standard model rather awkward.

The purpose of this paper is to modify the previous formalism [7] without introducing the extra discrete space for the strong interaction and to incorporate the color $\mathrm{SU}(3)$ gauge 
field(gluons) by affording the direct product internal symmetry

(semi-simple group) such as $\mathrm{SU}(3)_{c} \times \mathrm{SU}(2)_{L}$. The standard model needs $N=2$ discrete space for reconstruction in this formalism, while it needed $N=3$ discrete space in the previous formalism [6], [7]. LRSM is still alive as a model with the intermediate symmetry of the spontaneously broken $\mathrm{SO}(10)$ grand unified theory(GUT). $N=3$ discrete space is needed for the reconstruction of LRSM to include two Higgs bosons $\phi$ and $\xi$ which are as usual transformed as $\left(2,2^{*}, 0\right)$ and $(1,3,-2)$ under $\mathrm{SU}(2)_{L} \times \mathrm{SU}(2)_{R} \times \mathrm{U}(1)_{Y}$, respectively. $\xi$ is responsible for the first stage breakdown of symmetry to give rise to the seesaw mechanism and so make $\nu_{R}$ Majorana fermion . Up and down quarks have the different masses from each other through the vacuum expectation value of $\phi$ leaving $\mathrm{U}(1)_{e m}$ invariant.

This paper is divided into five sections. The next section presents the modifications

of our previous formalism based on the generalized differential calculus on $M_{4} \times Z_{N}$. The generalized gauge field is defined there and a geometrical picture for the unification of the gauge and Higgs fields is realized. The third section is the application to the standard model which leads to rather clear scheme compared with our previous one. The fourth section provides the reconstruction of LRSM. It will be realized that the boson and leptonic sectors are nicely incorporated so that the seesaw mechanism works well to give the right-handed neutrino huge mass and the left-handed neutrino extremely small mass. The last section is devoted for concluding remarks.

\section{Generalized gauge field with direct product form}

The generalized gauge field $A(x, n)$ in non-commutative geometry on the discrete space $M_{4} \times Z_{N}$ was given in Ref.[7] as

$$
A(x, n)=\sum_{i} a_{i}^{\dagger}(x, n) \mathbf{d} a_{i}(x, n)
$$


where $a_{i}(x, n)$ is the square-matrix-valued function and $\mathbf{d}$ is the generalized exterior derivative defined as follows.

$$
\begin{aligned}
& \mathbf{d} a_{i}(x, n)=\left(d+d_{\chi}\right) a(x, n)=\left(d+\sum_{k=1}^{N} d_{\chi_{k}}\right) a(x, n) \\
& d a_{i}(x, n)=\partial_{\mu} a_{i}(x, n) d x^{\mu} \\
& d_{\chi_{k}} a_{i}(x, n)=\left[-a_{i}(x, n) M_{n k}+M_{n k} a_{i}(x, k)\right] \chi_{k}
\end{aligned}
$$

Here $d x^{\mu}$ is ordinary one form basis, taken to be dimensionless, in $M_{4}$, and $\chi_{k}$ is the one form basis, assumed to be also dimensionless, in the discrete space $Z_{N}$ with the variable $n(n=1, \cdots, N)$. We have introduced $x$-independent matrix $M_{n m}$ whose hermitian conjugation is given by $M_{n k}^{\dagger}=M_{k n}$. The matrix $M_{n k}$ turns out to determine the scale and pattern of the spontaneous breakdown of the gauge symmetry. We here skip to explain detailed algebras with respect to non-commutative geometry because those are seen in Ref.[7]. According to Ref.[7], we can define the gauge fields $A_{\mu}(x, n)$ and the Higgs field $\Phi_{n m}(x)$ as

$$
\begin{aligned}
& A_{\mu}(x, n)=\sum_{i} a_{i}^{\dagger}(x, n) \partial_{\mu} a_{i}(x, n), \\
& \Phi_{n m}(x)=\sum_{i} a_{i}^{\dagger}(x, n)\left(-a_{i}(x, n) M_{n m}+M_{n m} a_{i}(x, m)\right) .
\end{aligned}
$$

We extend Eq.(1) to the generalized gauge field with the direct product form to incorporate gluon field on the same sheet as flavor gauge fields.

$$
\mathcal{A}(x, n)=\sum_{i} a_{i}^{\dagger}(x, n) \mathbf{d} a_{i}(x, n) \otimes 1+1 \otimes \sum_{j} b_{j}^{\dagger}(x, n) \mathbf{d} b_{j}(x, n)
$$

where the second term is responsible for the gluon field, so that actually $\mathbf{d} b_{j}(x, n)=$ $d b_{j}(x)$ because the strong interaction does not break down spontaneously. We assume $b_{j}(x, n)=b_{j}(x)$ which means the strong interaction works on the every discrete space $(n=1,2, \cdots, N)$. In the same context as in Eq.(3) $)$, the gluon field $G_{\mu}(x)$ is expressed as

$$
G_{\mu}(x)=\sum_{j} b_{j}^{\dagger}(x) \partial_{\mu} b_{j}(x)
$$


where $n$-independence of $G_{\mu}(x)$ means that the gluon field exists on the every sheet $(n=$ $1,2, \cdots N)$ as stated above. In order to identify $A_{\mu}(x, n)$ and $G_{\mu}(x)$ as true gauge fields, the following conditions have to be imposed.

$$
\begin{aligned}
& \sum_{i} a_{i}^{\dagger}(x, n) a_{i}(x, n)=1, \\
& \sum_{j} b_{j}^{\dagger}(x) b_{j}(x)=\frac{1}{g_{s}},
\end{aligned}
$$

where $g_{s}$ is a constant related to the corresponding coupling constant shown later. $i$ is a variable of the extra internal space which we can not now identify what it is. Eqs.(3) and (5) are very similar to the effective gauge field in Berry phase [10], which may lead to the identification of this internal space. In general, we can put the right hand side of the first equation in Eq.(6) to be $\frac{1}{g_{n}}$. However, we put it as it is to avoid the complexity.

Before constructing the gauge covariant field strength, we address the gauge transformation of $a_{i}(x, n)$ and $b_{j}(x)$ which is defined as

$$
\begin{aligned}
& a_{i}^{g}(x, n)=a_{i}(x, n) g(x, n), \\
& b_{j}^{g}(x)=b_{j}(x) g_{s}(x),
\end{aligned}
$$

where $g(x, n)$ and $g_{s}(x)$ are the gauge functions with respect to the corresponding flavor unitary group and the color $\mathrm{SU}(3)_{c}$ group, respectively. Then, we can get the gauge transformation of $\mathcal{A}(x, n)$ to be

$$
\begin{aligned}
\mathcal{A}^{g}(x, n)= & g^{-1}(x, n) \otimes g_{s}^{-1}(x) \mathcal{A}(x, n) g(x, n) \otimes g_{s}(x) \\
& +g^{-1}(x, n) \mathbf{d} g(x, n) \otimes 1+1 \otimes \frac{1}{g_{s}} g_{s}^{-1}(x) d g_{s}(x),
\end{aligned}
$$

where use has been made of Eq.(6) and as in Eq.(2),

$$
\begin{aligned}
\mathbf{d} g(x, n)=\partial_{\mu} g(x, n) d x^{\mu} & \\
& +\left[-g(x, n) M_{n k}+M_{n k} g(x, k)\right] \chi_{k} .
\end{aligned}
$$

Eq.(8) affords us to construct the gauge covariant field strength as follows:

$$
\mathcal{F}(x, n)=F(x, n) \times 1+1 \times \mathcal{G}(x),
$$


where $F(x, n)$ and $\mathcal{G}(x)$ are the field strengths of flavor and color gauge fields, respectively and given as

$$
\begin{aligned}
& F(x, n)=\mathbf{d} A(x, n)+A(x, n) \wedge A(x, n) \\
& \mathcal{G}(x)=d G(x)+g_{s} G(x) \wedge G(x) .
\end{aligned}
$$

The algebras of non-commutative differential geometry defined in Ref. [7] yields

$$
\begin{aligned}
F(x, n)= & \frac{1}{2} F_{\mu \nu}(x, n) d x^{\mu} \wedge d x^{\nu}+\sum_{m \neq n} D_{\mu} \Phi_{n m}(x) d x^{\mu} \wedge \chi_{m} \\
& +\sum_{k \neq n} V_{n k}(x) \chi_{k} \wedge \chi_{n} \\
& +\sum_{k \neq n} \sum_{\{m \neq k, n\}} V_{n k m}(x) \chi_{k} \wedge \chi_{m},
\end{aligned}
$$

where

$$
\begin{aligned}
& F_{\mu \nu}(x, n)=\partial_{\mu} A_{\nu}(x, n)-\partial_{\nu} A_{\mu}(x, n)+\left[A_{\mu}(x, n), A_{\mu}(x, n)\right], \\
& D_{\mu} \Phi_{n m}(x)=\partial_{\mu} \Phi_{n m}(x)-\left(\Phi_{n m}(x)+M_{n m}\right) A_{\mu}(x, m) \\
& +A_{\mu}(x, n)\left(M_{n m}+\Phi_{n m}(x)\right) \\
& V_{n k}(x)=\left(\Phi_{n k}(x)+M_{n k}\right)\left(\Phi_{k n}(x)+M_{k n}\right)-Y_{n k}(x), \quad \text { for } k \neq n \\
& V_{n k m}(x)=\left(\Phi_{n k}(x)+M_{n k}\right)\left(\Phi_{k m}(x)+M_{k m}\right)-Y_{n k m}(x) \text {. for } k \neq n, m \neq n
\end{aligned}
$$

$Y_{n k}(x, n)$ and $Y_{n l m}$ in Eq.(13) are auxiliary fields and expressed as

$$
\begin{aligned}
& Y_{n k}(x)=\sum_{i} a_{i}^{\dagger}(x, n) M_{n k} M_{k n} a_{i}(x, n), \quad \text { for } n \neq k, \\
& Y_{n k m}(x)=\sum_{i} a_{i}^{\dagger}(x, n) M_{n k} M_{k m} a_{i}(x, m) .
\end{aligned}
$$

If we define $H_{n m}(x)=\Phi_{n m}(x)+M_{n m}$, it is readily known that the function $H_{n m}(x)$ represents the unshifted Higgs field, whereas $\Phi_{n m}(x)$ denotes the shifted Higgs field with vanishing vacuum expectation value so that $M_{n m}$ determines the scale and pattern of the spontaneous breakdown of gauge symmetry. In contrast to $F(x, n), \mathcal{G}(x)$ is simply denoted as

$$
\begin{aligned}
\mathcal{G}(x) & =\frac{1}{2} \mathcal{G}_{\mu \nu}(x) d x^{\mu} \wedge d x^{\nu} \\
& =\frac{1}{2}\left\{\partial_{\mu} G_{\nu}(x)-\partial_{\nu} G_{\mu}(x)+g_{s}\left[G_{\mu}(x), G_{\mu}(x)\right]\right\} d x^{\mu} \wedge d x^{\nu}
\end{aligned}
$$


With the same metric structure on the discrete space $M_{4} \times Z_{N}$ as in Ref. [7] we can obtain the gauge invariant Yang-Mills-Higgs lagrangian (YMH)

$$
\begin{aligned}
& \mathcal{L}_{Y M H}(x)=-\sum_{n=1}^{N} \frac{1}{g_{n}^{2}}<\mathcal{F}(x, n), \mathcal{F}(x, n)> \\
&=-\operatorname{tr} \sum_{n=1}^{N} \frac{1}{2 g_{n}^{2}} F_{\mu \nu}^{\dagger}(x, n) F^{\mu \nu}(x, n) \\
&+\operatorname{tr} \sum_{n=1}^{N} \sum_{m \neq n} \frac{\alpha^{2}}{g_{n}^{2}}\left(D_{\mu} \Phi_{n m}(x)\right)^{\dagger} D^{\mu} \Phi_{n m}(x) \\
&-\operatorname{tr} \sum_{n=1}^{N} \frac{\alpha^{4}}{g_{n}^{2}} \sum_{k \neq n} V_{n k}^{\dagger}(x) V_{n k}(x)-\operatorname{tr} \sum_{n=1}^{N} \frac{\alpha^{4}}{g_{n}^{2}} \sum_{k \neq n} \sum_{m \neq n, k} V_{n k m}^{\dagger}(x) V_{n k m}(x), \\
&-\operatorname{tr} \sum_{n=1}^{N} \frac{1}{2 g_{n}^{2}} \mathcal{G}_{\mu \nu}^{\dagger}(x) \mathcal{G}^{\mu \nu}(x),
\end{aligned}
$$

where $g_{n}$ is a constant relating to the coupling constant of the flavor gauge field and tr denotes the trace over internal symmetry matrices. $\alpha$ emerges from the definition of metric $<\chi_{n}, \chi_{m}>=-\alpha^{2} \delta_{n m}$. The third term is the potential term of Higgs particle and the fourth term is the interaction term between Higgs particles.

Let us turn to the fermion sector to construct the Dirac Lagrangian. This is also deeply indebted to Ref. [7] so that only main points should be explained by skipping details. Let us start to define the covariant derivative acting on the spinor field $\psi(x, n)$ which is the representation of the corresponding semi simple group including $\mathrm{SU}(3)_{c}$.

$$
\mathcal{D} \psi(x, n)=\left(\mathbf{d}+\mathcal{A}^{f}(x, n)\right) \psi(x, n),
$$

which we call the covariant spinor one-form. The algebraic rules in Ref.[7] along with Eq.(四) leads Eq.(17) to

$$
\begin{aligned}
\mathcal{D} \psi(x, n)= & \left\{1 \otimes 1 \partial_{\mu}+\left(A_{\mu}^{f}(x, n) \otimes 1 d x^{\mu}+\sum_{m} H_{n m}(x) \otimes 1 \chi_{m}\right)\right. \\
& \left.+1 \otimes G_{\mu}^{f}(x) d x^{\mu}\right\} \psi(x, n),
\end{aligned}
$$

where $A_{\mu}^{f}(x, n)$ and $G_{\mu}^{f}(x)$ are the differential representations with respect to $\psi(x, n)$ and do not necessarily coincide with $A_{\mu}(x, n)$ and $G_{\mu}(x)$ in the gauge boson sector, respectively. It should be noticed that $\mathcal{D} \psi(x, n)$ is gauge covariant so that

$$
\mathcal{D} \psi^{g}(x, n)=\left(g^{f}(x, n)\right)^{-1} \otimes\left(g_{s}^{f}(x)\right)^{-1} \mathcal{D} \psi(x, n),
$$


where $g^{f}(x, n) \otimes g_{s}^{f}(x)$ is the gauge transformation function with respect to the representation of $\psi(x, n)$. Corresponding Eq.(17), the associated spinor one-form is introduced by

$$
\tilde{\mathcal{D}} \psi(x, n)=1 \otimes 1\left\{\gamma_{\mu} \psi(x, n) d x^{\mu}-i c_{Y} \psi(x, n) \sum_{m} \chi_{m}\right\}
$$

where $c_{Y}$ is a real dimensionless constant related to the Yukawa coupling constant between Higgs field and fermions. With the same inner products for spinor one-forms as in Ref. [7], we can get the Dirac Lagrangian.

$$
\begin{aligned}
\mathcal{L}_{D}(x, n)= & i<\tilde{\mathcal{D}} \psi(x, n), \mathcal{D} \psi(x, n)> \\
= & i \operatorname{tr}\left[\bar{\psi}(x, n) \gamma^{\mu}\left(1 \otimes 1 \partial_{\mu}+A_{\mu}^{f}(x, n) \otimes 1+1 \otimes G_{\mu}^{f}(x)\right) \psi(x, n)\right. \\
& \left.+i g_{Y} \bar{\psi}(x, n) \sum_{m} H_{n m}(x) \otimes 1 \psi(x, m)\right]
\end{aligned}
$$

where $c_{Y}$ is changed into $g_{Y}$ by appropriate replacement. The total Dirac Lagrangian is the sum over $n$ :

$$
\mathcal{L}_{D}(x)=\sum_{n=1}^{N} \mathcal{L}_{D}(x, n)
$$

which is apparently invariant for the Lorentz and gauge transformations. Eqs.(16) and (22) along with Eq. 21) are crucially important to reconstruct the spontaneously broken gauge theory.

With these preparations, we can apply the direct product formalism proposed in this section to the standard model and the left-right symmetric model, which make the presentations rather simpler than those before.

\section{Standard model}

Reconstruction of the standard model in non-commutative geometry on the discrete space has been already completed in Ref. [6], [7] where the extra sheet for the color gauge (gluon) field is arranged $(N=3)$, which made the presentation rather awkward. However, the 
present formulation which affords the incorporation of direct product gauge fields makes it clear, because it needs only two sheets $(N=2)$.

We identify $G_{\mu}(x)$ in Eq.(5) with the gluon field as

$$
G_{\mu}(x)=-\frac{i}{2} \sum_{a=1}^{8} \lambda^{a} G_{\mu}^{a}(x),
$$

where $\lambda^{a}(a=1,2, \cdots 8)$ are the Gell-Mann matrices and $A_{\mu}(x, n), \Phi_{n k}(x)$ and $M_{n k}$ $(n, m=1,2)$ as

$$
\begin{aligned}
& A_{\mu}(x, 1)=-\frac{i}{2} \sum_{i=1}^{3} \tau^{i} A_{\mu}^{i}(x)-\frac{i}{2} a \tau^{0} B_{\mu}(x), \\
& \Phi_{12}(x)=\left(\begin{array}{c}
\phi_{+}(x) \\
\phi_{0}(x)
\end{array}\right), \quad M_{12}=\left(\begin{array}{c}
0 \\
\mu
\end{array}\right),
\end{aligned}
$$

where $A_{\mu}^{i}(x)$ is $S U(2)$ gauge field and $B_{\mu}(x)$ is $U(1)$ gauge field, and

$$
\begin{aligned}
& A_{\mu}(x, 2)=-\frac{i}{2} b B_{\mu}(x), \\
& \Phi_{21}(x)=\Phi_{12}^{\dagger}(x), \quad M_{21}=(0, \mu)=M_{12}^{\dagger} .
\end{aligned}
$$

$\mu$ in $M_{n k}$ is a real, positive constant and there exist the free real parameters $a, b$ in Eqs.(24) and (25).

From these specifications, the generalized field strength $\mathcal{F}(x, n)$ is expressed as

$$
\begin{aligned}
\mathcal{F}(x, 1)= & -\frac{i}{4} \sum_{a} 1 \otimes \lambda^{a} \mathcal{G}_{\mu \nu}^{a}(x) d x^{\mu} \wedge d x^{\nu}, \\
& +\frac{i}{4}\left[-\sum_{i} \tau^{i} F_{\mu \nu}^{i}(x)-a \tau^{0} B_{\mu \nu}(x)\right] \otimes 1 d x^{\mu} \wedge d x^{\nu} \\
+ & D_{\mu} H(x) \otimes 1 d x^{\mu} \wedge \chi_{2}+\left[H(x) H^{\dagger}(x)-Y_{23}(x)\right] \otimes 1 \chi_{1} \wedge \chi_{2}, \\
F(x, 2)= & -\frac{i}{4} \sum_{a} 1 \otimes \lambda^{a} \mathcal{G}_{\mu \nu}^{a}(x) d x^{\mu} \wedge d x^{\nu}-b \frac{i}{4} B_{\mu \nu}(x) 1 \otimes 1 d x^{\mu} \wedge d x^{\nu} \\
& +\left(D_{\mu} H(x)\right)^{\dagger} \otimes 1 d x^{\mu} \wedge \chi_{1}+\left[H^{\dagger}(x) H(x)-Y_{32}(x)\right] \otimes 1 \chi_{2} \wedge \chi_{1},
\end{aligned}
$$

where

$$
\begin{gathered}
\mathcal{G}_{\mu \nu}^{a}(x)=\partial_{\mu} G_{\nu}^{a}(x)-\partial_{\nu} G_{\mu}^{a}(x)+g_{s} f^{a b c} G_{\mu}^{b}(x) G_{\nu}^{c}(x), \\
F_{\mu \nu}^{i}(x)=\partial_{\mu} A_{\nu}^{i}(x)-\partial_{\nu} A_{\mu}^{i}(x)+\epsilon^{i j k} A_{\mu}^{j}(x) A_{\nu}^{k}(x),
\end{gathered}
$$




$$
\begin{aligned}
& B_{\mu \nu}(x)=\partial_{\mu} B_{\nu}(x)-\partial_{\nu} B_{\mu}(x), \\
& H(x)=\Phi_{23}(x)+M_{23} . \\
& D^{\mu} H(x)=\left[\partial_{\mu}-\frac{i}{2}\left(\sum_{i} \tau^{i} A_{\mu}^{i}(x)+(a-b) \tau^{0} B_{\mu}(x)\right)\right] H(x) .
\end{aligned}
$$

From here we can proceed in the same way as in Ref. [7] including the handling of the auxiliary fields $Y_{n k}(x)$ and scale transformations of fields, so that we skip all of procedures to obtain YMH in the standard model (See Ref.[0]).

Let us turn to the fermionic sector. We can achieve the same results as in Ref.[7] by replacing $\psi(x, 2)$ and $\psi(x, 3)$ in Ref. [7] by $\psi(x, 1)$ and $\psi(x, 2)$, respectively. As for the leptons, they are singlet for the color gauge group, so that the differential representation $G_{\mu}^{f}(x)$ in Eq. (18) vanishes. We have the two kind of specifications $\psi(x, n)(n=1,2)$ for quark sector to give masses not only down quark but also up quark for which the differential representations do not vanish. We can carry all calculations in the same way as in Ref. [7] and get the same conclusions. Thus, we skip all detailed explanations about the fermionic sector.

\section{Left-right symmetric model}

The purpose of this section is to reconstruct the left-right symmetric gauge model (LRSM ) in our formalism. LRSM is the gauge theory with the symmetry $\mathrm{SU}(3)_{c} \times \mathrm{SU}(2)_{L} \times \mathrm{SU}(2)_{R} \times$ $\mathrm{U}(1)$ and still alive as a model with the intermediate symmetry of the spontaneously broken $\mathrm{SO}(10)$ GUT which is the most promising grand unified theory in particle physics. Thus, it is still worthwhile to reconstruct LRSM, and it is also important for the test of our formalism. Let us begin with the bosonic sector.

\section{A. Yang-Mills-Higgs sector}

In order to get YMH in LRSM we prepare $(N=3)$ discrete space with three sheets. Let us begin to specify $\mathcal{A}(x, n)(n=1,2,3)$.

$$
\mathcal{A}(x, 1)=A_{\mu}(x, 1) \otimes 1+1 \otimes G_{\mu}(x) d x^{\mu}+\Phi_{12}(x) \otimes 1 \chi_{2},
$$


where

$$
\begin{aligned}
& G_{\mu}(x)=-\frac{i}{2} \sum_{a=1}^{8} \lambda^{a} G_{\mu}^{a}(x) \\
& A_{\mu}(x, 1)=-\frac{i}{2} \sum_{k} \tau_{k} A_{L \mu}^{k}-\frac{i}{2} a \tau^{0} B_{\mu}=-\frac{i}{2}\left(\begin{array}{cc}
A_{L \mu}^{3}+a B_{\mu} & \sqrt{2} W_{L \mu} \\
\sqrt{2} W_{L \mu}^{\dagger} & -A_{L \mu}^{3}+a B_{\mu}
\end{array}\right),
\end{aligned}
$$

with the Pauli matrices $\tau_{k}(k=1,2,3), 2 \times 2$ unit matrix $\tau^{0}$ and $W_{L}=\left(A_{L}^{1}-i A_{L}^{2}\right) / \sqrt{2}$, and

$$
\begin{aligned}
& \Phi_{12}=\Phi_{21}^{\dagger}=\phi=\left(\begin{array}{cc}
\phi_{2}^{0} & \phi_{1}^{+} \\
\phi_{2}^{-} & \phi_{1}^{0}
\end{array}\right), \quad M_{12}=M_{21}^{\dagger}=\left(\begin{array}{cc}
\mu_{2} & 0 \\
0 & \mu_{1}
\end{array}\right) . \\
& \mathcal{A}(x, 2))=\left(A_{\mu}(x, 2) \otimes 1+1 \otimes G_{\mu}(x)\right) d x^{\mu}+\Phi_{21}(x) \otimes 1 \chi_{1}+\Phi_{23}(x) \otimes 1 \chi_{3},
\end{aligned}
$$

where

$$
A_{\mu}(x, 2)=-\frac{i}{2} \sum_{k} \tau_{k} A_{R \mu}^{k}-\frac{i}{2} b \tau^{0} B_{\mu}=-\frac{i}{2}\left(\begin{array}{cc}
A_{R \mu}^{3}+b B_{\mu} & \sqrt{2} W_{R \mu} \\
\sqrt{2} W_{R \mu}^{\dagger} & -A_{R}^{3}+b B_{\mu}
\end{array}\right),
$$

with $W_{R}=\left(A_{R}^{1}-i A_{R}^{2}\right) / \sqrt{2}$, and

$$
\begin{aligned}
& \Phi_{23}=\Phi_{32}^{\dagger}=\xi=\left(\begin{array}{cc}
\xi^{-} & \xi^{0} \\
\xi^{--} & -\xi^{-}
\end{array}\right), \quad M_{23}=M_{32}^{\dagger}=\left(\begin{array}{cc}
0 & M \\
0 & 0
\end{array}\right) . \\
& \mathcal{A}(x, 3)=\left(A_{\mu}(x, 3) \otimes 1+1 \otimes G_{\mu}(x)\right) d x^{\mu}+\Phi_{32}(x) \otimes \chi_{2},
\end{aligned}
$$

where

$$
A_{\mu}(x, 3)=-\frac{i}{2} \sum_{k} \tau_{k} A_{R \mu}^{k}-\frac{i}{2} c \tau^{0} B_{\mu}=-\frac{i}{2}\left(\begin{array}{cc}
A_{R \mu}^{3}+c B_{\mu} & \sqrt{2} W_{R \mu} \\
\sqrt{2} W_{R \mu}^{\dagger} & -A_{R \mu}^{3}+c B_{\mu}
\end{array}\right) .
$$

We assume $M_{13}=M_{31}^{\dagger}=0$ and so $\Phi_{13}=\Phi_{31}=0$ in the above specifications. There are three parameters $a, b$ and $c$ in above equations. However, $a=b$ is required because the hypercharge of $\phi$ is zero. We have to take gauge transformation functions as

$$
\begin{aligned}
& g(x, 1)=e^{-i a \alpha(x)} g_{L}(x), e^{-i a \alpha(x)} \in U(1), g_{L}(x) \in S U(2)_{L}, \\
& g(x, 2)=e^{-i b \alpha(x)} g_{R}(x), e^{-i b \alpha(x)} \in U(1), g_{R}(x) \in S U(2)_{R}, \\
& g(x, 3)=e^{-i c \alpha(x)} g_{R}(x), e^{-i c \alpha(x)} \in U(1), g_{R}(x) \in S U(2)_{R} .
\end{aligned}
$$


$\phi$ and $\xi$ transform under $\mathrm{SU}(2)_{L} \times \mathrm{SU}(2)_{R} \times \mathrm{U}(1)$ according to $\left(2,2^{*}, 0\right)$ and $(1,3,-2)$ representations, respectively because of $a=b=-c$.

With these assignments we can calculate Yang-Mills-Higgs lagrangian $\mathcal{L}_{Y M H}(x)$ in Eq.(16). $\mathcal{L}_{Y M H}(x)$ is denoted as

$$
\mathcal{L}_{Y M H}(x)=\mathcal{L}_{G B}+\mathcal{L}_{H K}-V_{H P},
$$

where $\mathcal{L}_{G B}, \mathcal{L}_{H K}$ and $V_{H P}$ are the gauge boson kinetic term, the Higgs boson kinetic term, and the Higgs boson potential term respectively.

$$
\begin{aligned}
\mathcal{L}_{G B}= & -\frac{1}{4}\left(\sum_{i=1}^{3} \frac{1}{g_{i}^{2}}\right) \sum_{a} G_{\mu \nu}^{a} G^{a \mu \nu} \\
& -\frac{1}{4} \frac{1}{g_{1}^{2}} \sum_{i} F_{L \mu \nu}^{i} F_{L}^{i \mu \nu}-\frac{1}{4}\left(\frac{1}{g_{2}^{2}}+\frac{1}{g_{3}^{3}}\right) \sum_{i} F_{R \mu \nu}^{i} F_{R}^{i \mu \nu} \\
& -\frac{1}{4}\left(\frac{a^{2}}{g_{1}^{2}}+\frac{b^{2}}{g_{2}^{2}}+\frac{c^{2}}{g_{3}^{2}}\right) B_{\mu \nu} B^{\mu \nu} .
\end{aligned}
$$

By denoting $g_{0}=\left(\sum_{i=1}^{3} \frac{1}{g_{i}^{2}}\right)^{-\frac{1}{2}}, g_{L}=g_{1}, g_{R}=\left(\frac{1}{g_{2}^{2}}+\frac{1}{g_{3}^{2}}\right)^{-\frac{1}{2}}$ and $g_{B}=\left(\frac{a^{2}}{g_{1}^{2}}+\frac{b^{2}}{g_{2}^{2}}+\frac{c^{2}}{g_{3}^{2}}\right)^{-\frac{1}{2}}$, and replacing $G_{\mu}^{a}, A_{L \mu}, A_{R \mu}$ and $B_{\mu}$ by $g_{0} G_{\mu}^{a}, g_{L} A_{L \mu}, g_{R} A_{R \mu}$ and $g_{B} B_{\mu}$, respectively we can find the standard gauge boson kinetic term as

$$
\begin{aligned}
\mathcal{L}_{G B}= & -\frac{1}{4} \sum_{a} G_{\mu \nu}^{a} G^{a \mu \nu} \\
& -\frac{1}{4} \sum_{i} F_{L \mu \nu}^{i} F_{L}^{i \mu \nu}-\frac{1}{4} \sum_{i} F_{R}^{i}{ }_{R \nu} F_{R}^{i \mu \nu}-\frac{1}{4} B_{\mu \nu} B^{\mu \nu},
\end{aligned}
$$

where

$$
\begin{aligned}
& G_{\mu \nu}^{a}=\partial_{\mu} G_{\nu}^{a}-\partial_{\nu} G_{\mu}^{a}+g_{s} f^{a b c} G_{\mu}^{b} G_{\nu}^{c}, \\
& F_{(L, R) \mu \nu}^{i}=\partial_{\mu} A_{(L, R) \nu}^{i}-\partial_{\nu} A_{(L, R) \mu}^{i}+g_{L, R} \epsilon^{i j k} A_{(L, R) \mu}^{j} A_{(L, R) \nu}^{k}, \\
& B_{\mu \nu}=\partial_{\mu} B_{\nu}-\partial_{\nu} B_{\mu},
\end{aligned}
$$

with the replacement $g_{0} g_{s} \rightarrow g_{s}$.

Higgs kinetic term $\mathcal{L}_{H K}$ is written as follows:

$$
\mathcal{L}_{H K}=\operatorname{Tr} \mid \partial_{\mu} \phi-\frac{i}{2}\left(g_{L} \sum_{k=1}^{3} \tau_{k} A_{L \mu}^{k}+a g_{B} B_{\mu} \tau_{0}\right)\left(\phi+M_{12}\right)
$$




$$
\begin{array}{r}
+\left.\frac{i}{2}\left(\phi+M_{12}\right)\left(g_{R} \sum_{k=1}^{3} \tau_{k} A_{R \mu}^{k}+b g_{B} B_{\mu} \tau_{0}\right)\right|^{2} \\
+\operatorname{Tr} \mid \partial_{\mu} \xi-\frac{i}{2}\left(g_{R} \sum_{k=1}^{3} \tau_{k} A_{R \mu}^{k}+b g_{B} B_{\mu} \tau_{0}\right)\left(\xi+M_{23}\right) \\
+\left.\frac{i}{2}\left(\xi+M_{23}\right)\left(g_{R} \sum_{k=1}^{3} \tau_{k} A_{R \mu}^{k}+c g_{B} B_{\mu} \tau_{0}\right)\right|^{2}
\end{array}
$$

where the replacements

$$
\begin{array}{ll}
\left(\frac{\alpha^{2}}{g_{L}^{2}}+\frac{\alpha^{2}}{g_{2}^{2}}\right)^{\frac{1}{2}} \phi \rightarrow \phi, & \left(\frac{\alpha^{2}}{g_{L}^{2}}+\frac{\alpha^{2}}{g_{2}^{2}}\right)^{\frac{1}{2}} M_{12} \rightarrow M_{12}, \\
\left(\frac{\alpha^{2}}{g_{2}^{2}}+\frac{\alpha^{2}}{g_{3}^{2}}\right)^{\frac{1}{2}} \xi \rightarrow \xi, \quad\left(\frac{\alpha^{2}}{g_{2}^{2}}+\frac{\alpha^{2}}{g_{3}^{2}}\right)^{\frac{1}{2}} M_{23} \rightarrow M_{23},
\end{array}
$$

have been done.

Let us move to the Higgs potential terms. In order to avoid the complexity in expressions, $g_{1}=g_{2}=g_{3}=g$ is assumed without loss of essence.

$$
\begin{aligned}
V_{H P} & =\frac{g^{2}}{4} \operatorname{Tr}\left|\left(\phi+M_{12}\right)\left(\phi^{\dagger}+M_{21}\right)-Y_{121}\right|^{2}+\frac{g^{2}}{4} \operatorname{Tr}\left|\left(\phi^{\dagger}+M_{21}\right)\left(\phi+M_{12}\right)-Y_{212}\right|^{2} \\
& +\frac{g^{2}}{4} \operatorname{Tr}\left|\left(\xi+M_{23}\right)\left(\xi^{\dagger}+M_{32}\right)-Y_{232}\right|^{2}+\frac{g^{2}}{4} \operatorname{Tr}\left|\left(\xi^{\dagger}+M_{32}\right)\left(\xi+M_{23}\right)-Y_{323}\right|^{2} \\
& +\frac{g^{2}}{2} \operatorname{Tr}\left|\left(\phi+M_{12}\right)\left(\xi+M_{23}\right)-Y_{123}\right|^{2}
\end{aligned}
$$

where the auxiliary fields $Y_{121}, Y_{323}$ and $Y_{123}$ are independent fields so that the terms containing these fields in Eq.(44) vanish due to the equation of motion, however,

$$
\begin{aligned}
& Y_{212}=\sum_{i} a_{i}^{\dagger}(2)\left(\begin{array}{cc}
\mu_{2}^{2} & 0 \\
0 & \mu_{1}^{2}
\end{array}\right) a_{i}(2), \\
& Y_{232}=\sum_{i} a_{i}^{\dagger}(2)\left(\begin{array}{cc}
M^{2} & 0 \\
0 & 0
\end{array}\right) a_{i}(2),
\end{aligned}
$$

have the relation that $M^{2}\left(Y_{212}-\mu_{1}^{2}\right)=\left(\mu_{2}^{2}-\mu_{1}^{2}\right) Y_{232}$ so that Eq.(44) leads to

$$
\begin{array}{r}
V_{H P}=\frac{g^{2}}{2} \frac{1}{M^{4}+\left(\mu_{1}^{2}-\mu_{2}^{2}\right)^{2}} \operatorname{Tr} \mid M^{2}\left\{\left(\phi^{\dagger}+M_{21}\right)\left(\phi+M_{12}\right)-M_{21} M_{12}\right\} \\
+\left.\left(\mu_{1}^{2}-\mu_{2}^{2}\right)\left\{\left(\xi+M_{23}\right)\left(\xi^{\dagger}+M_{32}\right)-M_{23} M_{32}\right\}\right|^{2} .
\end{array}
$$

The present formulation in non-commutative geometry is very restrictive to the Higgs potential and Higgs interacting terms. Eq. (46) includes those terms in more restrictive 
way. We think Eq.(46) to be taken at a specially chosen renormalization point. If $\mu_{1}>\mu_{2}$, we can chose the true vacuum at a point that the vacuum expectation values of $\phi$ and $\xi$ are zero, where the Higgs potential gets the minimum value so that the spontaneous symmetry breakdown takes place.

Gauge boson mass matrix can be extracted from Eq.(42). Thinking of the neutral gauge boson in the flavor sector, the remaining gauge symmetry is only $\mathrm{U}(1)_{e m}$, which should leads to the vanishing determinant of the neutral gauge boson mass matrix. Also for this purpose we have to take the relation $a=b$ which is due to the vanishing hypercharge of $\phi$. As a result the following mass matrix for the neutral gauge boson follows.

$$
M_{N G B}=\left(\begin{array}{ccc}
g_{L}^{2}\left(\mu_{1}^{2}+\mu_{2}^{2}\right) & -g_{L} g_{R}\left(\mu_{1}^{2}+\mu_{2}^{2}\right) & 0 \\
-g_{L} g_{R}\left(\mu_{1}^{2}+\mu_{2}^{2}\right) & g_{R}^{2}\left(\mu_{1}^{2}+\mu_{2}^{2}+4 M^{2}\right) & 2 g_{R} g_{B}(a-c) M^{2} \\
0 & 2 g_{R} g_{B}(a-c) M^{2} & g_{B}^{2}(a-c)^{2} M^{2}
\end{array}\right),
$$

which surely yields the vanishing determinant. On the other hand, charged gauge boson mass matrix is given also from Eq.(42) as

$$
M_{C G B}=\frac{1}{2}\left(\begin{array}{cc}
g_{L}^{2}\left(\mu_{1}^{2}+\mu_{2}^{2}\right) & -2 g_{L} g_{R} \mu_{1} \mu_{2} \\
-2 g_{L} g_{R} \mu_{1} \mu_{2} & g_{R}^{2}\left(\mu_{1}^{2}+\mu_{2}^{2}+M^{2}\right)
\end{array}\right) \text {. }
$$

Equations (47) and (48) enable us to estimate the gauge boson masses in the limit of $M \gg \mu_{1}, \mu_{2}$. According to Eq.(47), the neutral gauge boson masses are written as

$$
\begin{aligned}
& M_{\gamma}^{2}=0 \\
& M_{Z}^{2}=\left(g_{R}^{2}+g_{L}^{2}\right)\left(1+\frac{1}{2} \frac{\left((a-c)^{2} g_{B}^{2}-4 g_{R}^{2}\right)}{4 g_{R}^{2}+(a-c)^{2} g_{B}^{2}}\right)\left(\mu_{1}^{2}+\mu_{2}^{2}\right), \\
& M_{Z_{R}}^{2}=\left(4 g_{R}^{2}+(a-c)^{2} g_{B}^{2}\right) M^{2}
\end{aligned}
$$

where $\gamma$ and $Z$ represent of course photon and neutral weak boson, respectively and $Z_{R}$ denotes the extra neutral gauge boson expected in this model. $M_{Z_{R}}$ is estimated to be so large that one can not detect $Z_{R}$ in the energy range of the accelerator available nowadays.

From Eq.41) we can find mass matrix of the charged gauge boson masses as

$$
M_{W}^{2}=\frac{1}{2} g_{L}^{2}\left(\mu_{1}^{2}+\mu_{2}^{2}\right), \quad M_{W_{R}}^{2}=\frac{1}{2} g_{R}^{2}\left(\mu_{1}^{2}+\mu_{2}^{2}+2 M^{2}\right),
$$


where $W$ denotes the charged weak boson and $W_{R}$ is also the extra charged gauge boson expected in this model and its mass is so high that it can not be detectable.

If we assume $g_{L}^{2}=g_{R}^{2}=g$, the Weinberg angle is determined by

$$
\sin ^{2} \theta_{W}=1-\frac{M_{W}^{2}}{M_{Z}^{2}}=\frac{\mu_{2}^{2}}{\mu_{1}^{2}+\mu_{2}^{2}} .
$$

\section{B. Leptonic sector}

It should be noted that the leptonic sector must be well designed so that the seesaw mechanism nicely works to give the right-handed neutrino the huge mass and the lefthanded neutrino the extremely small mass [12]. $\Psi(x, n)$ is identified for each sheet $n=$ 1,2,3 as follows:

$$
\begin{array}{ll}
\psi(x, 1)=\frac{1}{\sqrt{2}} l_{L}=\frac{1}{\sqrt{2}}\left(\begin{array}{c}
\nu_{L} \\
e_{L}
\end{array}\right), & Y=-1, \\
\psi(x, 2)=\frac{1}{\sqrt{3}} l_{R}=\frac{1}{\sqrt{3}}\left(\begin{array}{c}
\nu_{R} \\
e_{R}
\end{array}\right), & Y=-1, \\
\psi(x, 3)=\frac{1}{\sqrt{3}} l_{R}^{c}=\frac{1}{\sqrt{3}}\left(\begin{array}{c}
e_{R}^{c} \\
-\nu_{R}^{c}
\end{array}\right), & Y=1,
\end{array}
$$

where the superscript $c$ denotes the charge conjugation of $e_{R}$ and $\nu_{R}$. The Nishijima-GellMann law $Q=T_{L}^{3}+T_{R}^{3}+Y / 2$ well works in Eq.(52).

From Eqs.(19),(20) and (52), we determine the covariant spinor one form $\mathcal{D} \psi(x, n)$ and the associated spinor one form $\tilde{\mathcal{D}} \psi(x, n)$ to obtain the Dirac Lagrangian for lepton sector, where it should be noticed that the differential representation $G^{f}(x)$ vanishes and $a(=b)$ and $c$ are identified with the hypercharges of the corresponding fermions.

$$
\begin{aligned}
\mathcal{D} \psi(x, 1)= & \left\{\partial_{\mu}-\frac{i}{2}\left(g_{L} \sum_{k} \tau_{k} A_{L \mu}^{k}-g_{B} \tau^{0} B_{\mu}\right)\right\} \psi(1) d x^{\mu} \\
& +g_{\phi}\left(\phi+M_{12}\right) \psi(2) \chi_{2} \\
\tilde{\mathcal{D}} \psi(x, 1)= & \gamma_{\mu} \psi(1) d x^{\mu}+i c_{l} \psi(1) \sum_{m \neq 1} \chi_{m}, \\
\mathcal{D} \psi(x, 2)= & \left\{\partial_{\mu}-\frac{i}{2}\left(g_{R} \sum_{k} \tau_{k} A_{R \mu}^{k}-g_{B} \tau^{0} B_{\mu}\right)\right\} \psi(2) d x^{\mu} \\
& +g_{\phi}\left(\phi^{\dagger}+M_{21}\right) \psi(1) \chi_{1}+g_{\xi}\left(\xi+M_{23}\right) \psi(3) \chi_{3}, \\
\tilde{\mathcal{D}} \psi(x, 2)= & \gamma_{\mu} \psi(2) d x^{\mu}+i c_{l} \psi(2) \sum_{m \neq 2} \chi_{m},
\end{aligned}
$$




$$
\begin{aligned}
\mathcal{D} \psi(x, 3)= & \left\{\partial_{\mu}-\frac{i}{2}\left(g_{R} \sum_{k} \tau_{k} A_{R \mu}^{k}+g_{B} \tau^{0} B_{\mu}\right)\right\} \psi(3) d x^{\mu} \\
& +g_{\xi}\left(\xi^{\dagger}+M_{32}\right) \psi(2) \chi_{2}, \\
\tilde{\mathcal{D}} \psi(x, 3)= & \gamma_{\mu} \psi(3) d x^{\mu}+i c_{l} \psi(3) \sum_{m \neq 3} \chi_{m} .
\end{aligned}
$$

From these equations together with Eqs.(21) and (22) we can get the Dirac Lagrangian for lepton sector.

$$
\begin{aligned}
\mathcal{L}_{D}= & i \bar{l}_{L} \gamma^{\mu}\left\{\partial_{\mu}-\frac{i}{2}\left(g_{L} \sum_{k} \tau_{k} A_{L \mu}^{k}-g_{B} \tau^{0} B_{\mu}\right)\right\} l_{L} \\
& +i \bar{l}_{R} \gamma^{\mu}\left\{\partial_{\mu}-\frac{i}{2}\left(g_{R} \sum_{k} \tau_{k} A_{R \mu}^{k}-g_{B} \tau^{0} B_{\mu}\right)\right\} l_{R} \\
& -g_{\phi}\left\{\bar{l}_{L}\left(\phi+M_{12}\right) l_{R}+\bar{l}_{R}\left(\phi^{\dagger}+M_{21}\right) l_{L}\right\} \\
& -g_{\xi}\left\{\bar{l}_{R}\left(\xi+M_{23}\right) l_{R}^{c}+\bar{l}_{R}^{c}\left(\xi^{\dagger}+M_{32}\right) l_{R}\right\},
\end{aligned}
$$

where the Yukawa coupling constants are changed in scale appropriately. Let us put attention on the mass terms in Eq.(56).

$$
\mathcal{L}_{\text {lepton mass }}=-\mu_{1}\left(\bar{e}_{R} e_{L}+\bar{e}_{L} e_{R}\right)-\mu_{2}\left(\bar{\nu}_{R} \nu_{L}+\bar{\nu}_{L} \nu_{R}\right)+M\left(\bar{\nu}_{R}^{c} \nu_{R}+\bar{\nu}_{R} \nu_{R}^{c}\right),
$$

where we neglect the coupling constants without loss of generality and leads to the neutrino mass matrix

$$
m_{\nu}=\left(\begin{array}{cc}
0 & \mu_{2} \\
\mu_{2} & -M
\end{array}\right)
$$

which is sufficient to give rise to the seesaw mechanism. - sign of one eigenvalue of matrix $m_{\nu}$ is absorbed into the phase of the wave function.

\section{Quark sector}

In contrast to lepton, quark interacts with gluon via the strong interaction and the mixing among three generations is conspicuous in quark sector. It is not so difficult to taken into account the quark mixing, which prescriptions have been already exhibited in Ref.[7]. Thus, for the sake of brevity, we consider here only one generation. $\psi(x, n)$ is 
identified for each sheet $n=1,2,3$ as follows:

$$
\begin{array}{ll}
\psi(x, 1)=\frac{1}{\sqrt{2}} q_{L}=\frac{1}{\sqrt{2}}\left(\begin{array}{l}
u_{L} \\
d_{L}
\end{array}\right), & Y=\frac{1}{3}, \\
\psi(x, 2)=\frac{1}{\sqrt{2}} q_{R}=\frac{1}{\sqrt{2}}\left(\begin{array}{l}
u_{R} \\
d_{R}
\end{array}\right), & Y=\frac{1}{3}, \\
\psi(x, 3)=0, &
\end{array}
$$

where we abbreviate subscripts about triplet representation of $S U(3)_{c} . \quad \psi(x, 3)$ have to be chosen to be zero owing to the respect of $\mathrm{U}(1)$ gauge transformation. $\psi(x, 1)$ are transformed as $\left(3,2,0, \frac{1}{3}\right)$ under $\mathrm{SU}(3)_{c} \times \mathrm{SU}(2)_{L} \times \mathrm{SU}(2)_{R} \times \mathrm{U}(1)$ and $\psi(x, 2)$ as $\left(3,0,2, \frac{1}{3}\right)$. As a result $\mathcal{D} \psi(x, n)$ and $\tilde{\mathcal{D}} \psi(x, n)$ are determined to be

$$
\begin{aligned}
\mathcal{D} \psi(x, 1)= & \left\{1 \otimes 1 \partial_{\mu}-\frac{i}{2} g_{s} \sum_{a=1}^{8} 1 \otimes \lambda_{a} G_{\mu}^{a}-\frac{i}{2}\left(g_{L} \sum_{k} \tau_{k} \otimes 1 A_{L \mu}^{k}\right.\right. \\
& \left.\left.+\frac{1}{3} g_{B} \tau^{0} \otimes 1 B_{\mu}\right)\right\} \psi(1) d x^{\mu}+g_{\phi}\left(\phi+M_{12}\right) \otimes 1 \psi(2) \chi_{2} \\
\tilde{\mathcal{D}} \psi(x, 1)= & \gamma_{\mu} \psi(1) d x^{\mu}+i c_{q} \psi(1) \sum_{m \neq 1} \chi_{m}, \\
\mathcal{D} \psi(x, 2)= & \left\{1 \otimes 1 \partial_{\mu}-\frac{i}{2} g_{s} \sum_{a=1}^{8} 1 \otimes \lambda_{a} G_{\mu}^{a}-\frac{i}{2}\left(g_{R} \sum_{k} \tau_{k} \otimes 1 A_{R \mu}^{k}\right.\right. \\
& \left.\left.+\frac{1}{3} g_{B} \tau^{0} \otimes 1 B_{\mu}\right)\right\} \psi(2) d x^{\mu}+g_{\phi}\left(\phi^{\dagger}+M_{21}\right) \otimes 1 \psi(1) \chi_{1}, \\
\tilde{\mathcal{D}} \psi(x, 2)= & \gamma_{\mu} \psi(2) d x^{\mu}+i c_{q} \psi(2) \sum_{m \neq 2} \chi_{m},
\end{aligned}
$$

From these equations we can obtain the Dirac Lagrangian for quark sector in first generation as follows:

$$
\mathcal{L}^{q}=\mathcal{L}_{\text {kin }}^{q}+\mathcal{L}_{\text {Yukawa }}^{q}
$$

where

$$
\begin{aligned}
\mathcal{L}_{k i n}^{q} & =i \bar{q}_{L} \gamma^{\mu}\left(\partial_{\mu}-\frac{i}{2} g_{s} \sum_{a=1}^{8} \lambda_{a} G_{\mu}^{a}-\frac{i}{2} g_{L} \sum_{k=1}^{3} \tau_{k} A_{L \mu}^{k}-\frac{i}{6} g_{B} \tau^{0} B_{\mu}\right) q_{L} \\
& +i \bar{q}_{R} \gamma^{\mu}\left(\partial_{\mu}-\frac{i}{2} g_{s} \sum_{a=1}^{8} \lambda_{a} G_{\mu}^{a}-\frac{i}{2} g_{R} \sum_{k=1}^{3} \tau_{k} A_{R \mu}^{k}-\frac{i}{6} g_{B} \tau^{0} B_{\mu}\right) q_{R}
\end{aligned}
$$

and

$$
\mathcal{L}_{\text {Yukawa }}^{q}=-g_{\phi}^{q}\left\{\bar{q}_{L}\left(\phi+M_{12}\right) q_{R}+\bar{q}_{R}\left(\phi^{\dagger}+M_{21}\right) q_{L}\right\}
$$


with the appropriately denoted coupling constants. Eq.(64) yields quark masses that $m_{u}=g_{\phi}^{q} \mu_{2}$ for up-quark and $m_{d}=g_{\phi}^{q} \mu_{1}$ for down-quark.

\section{Concluding remarks}

We have modified the scheme previously proposed by the present authors to be able to incorporate the color gauge field(gluon) without the extra discrete space for it. The modified scheme can afford the direct product gauge group (semi-simple group) such as $\mathrm{SU}(3)_{c} \times \mathrm{SU}(2)_{L, R}$ so that the strong interaction works in every sheet $(n=1,2, \cdots . N)$ of the discrete space $M_{4} \times Z_{N}$. It should be noticed that Lagrangian becomes meaningful after being summed over $n$ because such a procedure makes it Hermitian, and so we can not detect the discrete space $Z_{N}$ experimentally.

Then, we have constructed the standard model and the left-right symmetric gauge theory in non-commutative geometry using the formalism developed here. The reconstruction of the standard model need only $N=2$ discrete space, which makes it rather clearer compared to the previous one [6], [7]. LRSM is still alive as a model with the intermediate symmetry of the spontaneously broken $\mathrm{SO}(10)$ GUT which is the most promising unified theory in particle physics. Thus, whether we can actually reconstruct LRSM is an important step to achieve the justification of our formalism. We could nicely explain the seesaw mechanism to make the right-handed neutrino $\nu_{N}$ Majorana fermion and up and down quarks are given the different masses from each other. These are the necessary conditions for LRSM to be physically meaningful model. The only controversial point in our reconstruction of LRSM is about the Higgs potential. In general, the present formulation imposes the severe constraints on Higgs potential and interacting terms responsible for the symmetry breakdown. These constraints worked very well in the reconstruction of SU(5) GUT owing to the symmetry breakdown due to adjoint and $\mathbf{5}$ Higgs fields in $\mathrm{SU}(5)$ representation to result in the nice forms of Higgs potential and interacting terms [7]. Eq.(46) includes Higgs potential in more restrictive way. In this point we should un- 
derstand that our scheme in NCG gives Yang-Mills-Higgs lagrangian at a specially chosen renormalization point so that Eq.(46) takes the special form. Even so we can chose the true vacuum in Eq.(46) at a point that the vacuum expectation values of $\phi$ and $\xi$ are zero, where the Higgs potential gets the minimum value. Therefore, the spontaneous breakdown of symmetry can take place in the proper way.

We may discuss the evolution of the coupling constants in the Higgs potential in Eq. (46) by use of the renormalization equation. This subject including the quantization of the gauge theory with the restricted Higgs potential due to the non-commutative geometry will be studied in the future work.

\section{Acknowledgements}

One of the authors(Y.O.) would like to express his sincere thanks to Professor Y. Taka-

hashi and Professor H. Umezawa for hospitality at University of Alberta. This work was completed while Y.O. stayed at University of Alberta. 
REFERENCES

\section{References}

[1] A. Connes, p.9 in The Interface of Mathematics and Particle Physics, ed. D. G. Quillen, G. B. Segal, and Tsou. S. T., Clarendon Press, Oxford, 1990. See also, Alain Connes and J. Lott, Nucl. Phys. B(Proc. Suppl.) 18B, 57(1990).

[2] A. H. Chamseddine, G. Felder and J. Frölich, Phys. Lett. B296, 109(1992); Nucl. Phys. B395, 672(1993); A. H. Chamseddine and J. Frölich, Phys. Rev. D 50, 2893(1994); D. Kastler, Rev. Math. Phys. 5,477(1993); M. Dubois-Violette, Class. Quantum. Grav. 6,1709(1989); R. Coquereaux, G. Esposito-Farese, and G. Vaillant, Nucl. Phys. B353, 689(1991); M. Dubois-Violette, R. Kerner, and J. Madore, J. Math. Phys. 31, 316(1990); B. Balakrishna, F. Gürsey and K.C. Wali, Phys. Lett. B254,430(1991); Phys. Rev. D 46,6498(1992); R. Coquereaux, G. Esposito-Farese and F. Scheck, Int. Journ. Mod. Phys. A7, 6555(1992); R. Coquereaux, R. Haussling, N. Papadopoulos and F. Scheck, ibit. 7,2809(1992).

[3] A. Sitarz, Phys. Lett. , B308, 311(1993).

Jour. Geom. Phys. 15(1995), 123.

[4] H-G. Ding, H-Y. Gou, J-M. Li and K. Wu, preprint, ASITP-93-23, CCAST-93-5, “ Higgs as Gauge Fields on Discrete Groups and Standard Models for Electroweak and Electroweak-Strong Interactions ".

[5] B. Chen and K. Wu, preprint, AS-ITP-93-64, " $Z_{2} \odot Z_{2}$ Discrete Symmetry in the Left-Right Symmetric Model $S U(2)_{L} \times S U(2)_{R} \times U(1)_{B-L} "$.

[6] K. Morita and Y. Okumura, Phys. Rev. D 50, 1016(1994). Y. Okumura, Prog. Theor. Phys. ,92, 625(1994).

[7] K. Morita and Y. Okumura, Prog. Theor. Phys. 91, 975(1994). Y. Okumura, Phys. Rev. D 50, (1994) 1026. 
[8] K. Morita and Y. Okumura, Prog. Theor. Phys. 91, 959(1994).

[9] S. Naka and E. Umezawa, Prog. Theor. Phys. 92, 189 (1994)

[10] M.V. Berry, Proc. R. Soc. Lond. A392, 45(1984).

[11] Y. Okumura, Preprint, " SO(10) grand unified theory in non-commutative differential geometry on the discrete space $M_{4} \times Z_{N}{ }^{\prime \prime}$

[12] M. Gell-Mann, P. Ramond and R. Slanski, in Supergravity, Proceeding of the Workshop, Stony Brook, New-York, 1979, edited by P. van Nieuwenhuizen and D. Freedman(North-Holland, Amsterdam, 1980); T. Yanagida, in Proceeding of the Workshop on Unified Theories and Baryon Number in the Universe, Tsukuba, Japan, 1979,edited by A. Sawada and A. Sugamoto(KEK Report No.79-18 Tsukuba, 1979); R. N. Mohapartra and G. Senjanovic, Phys. Rev. Lett. 44,912(1980) and the references in M. Fukugita and T. Yanagida, Physics of Neutrinos in Physics and Astrophysics of Neutrinos, edited by M. Fukugita and A. Suzuki, Springer-Verlag, Tokyo, 1994. 